\title{
GESTIÓN TECNOLÓGICA EN EMPRESAS INNOVADORAS MEXICANAS
}

\section{Enrique Alberto Medellín Cabrera.}

Doctor en Ciencias de la Administración.

Facultad de Contaduría y Administración de la Universidad Nacional Autónoma de México (UNAM). eamc@cablevision.net.mx

\section{RESUMEN}

En este trabajo se revisa la experiencia en gestión tecnológica de 25 empresas innovadoras mexicanas, de diversos tamaños y sectores, ganadoras del Premio Nacional de Tecnología (PNT) entre 1999 y 2007. El objetivo es analizar los rasgos relevantes así como las principales prácticas de gestión de tecnología que reportan las empresas. Se llevó a cabo un estudio exploratorio utilizando información recogida por el PNT, completado con entrevistas a gerentes de diversas empresas e información divulgada por las mismas. Las actividades de innovación que se llevan a cabo en las empresas analizadas están acordes con las características de empresas innovadoras reportadas en la literatura especializada sobre el tema; y revelan que en su operación y actividades de innovación cumplen con los rasgos relevantes de gestión de tecnología que el autor identificó en el análisis de dicha literatura. Se identificaron dos grupos de rasgos relevantes: el primero soporta la creación de condiciones para la innovación en las empresas, y el segundo tiene que ver con un conjunto de prácticas de gestión de tecnología que juegan un rol importante en los procesos de innovación. Ambos grupos de rasgos distintivos son atendidos, aunque de diversas maneras, por las empresas que hacen de la innovación una de sus principales características para competir.

Palabras clave: Gestión de tecnologia; Empresas innovadoras; Características distintivas; México. 


\section{INTRODUCCIÓN}

La gestión de tecnología es una de las disciplinas que en las pasadas tres décadas más se ha ocupado de estudiar y desarrollar la innovación empresarial, y la innovación tecnológica en particular. Se ha ocupado de la comprensión del fenómeno innovador, de su modelaje, de sus prácticas, de sus impactos en la competitividad empresarial, de sus actores, de sus procesos y prácticas, de la interacción de estos últimos con otros procesos y prácticas organizacionales, de su medición y evaluación, de sus técnicas y herramientas, y de las condiciones externas e internas necesarias para que sea efectiva en un contexto y un momento histórico particular.

Para lograr lo anterior, los directivos y empleados que forman las empresas han debido comprender, entre otros factores, la naturaleza de las tecnologías que utilizan, las implicaciones que para sus negocios tienen las innovaciones tecnológicas, el tipo de respuestas estratégicas y operacionales a poner en práctica, los desafíos organizacionales que plantean los cambios tecnológicos, y los requerimientos para poder competir en mercados cada vez más exigentes y dinámicos. Estos retos, situaciones y respuestas empresariales conforman el sustrato práctico y teórico de lo que se ha denominado la gestión de tecnología. Estos factores, que se comentan en este trabajo, forman parte de los rasgos que diversos autores han considerado relevantes de la gestión de tecnología.

Con la gestión de tecnología: a) las empresas buscan maximizar sus ventajas competitivas, basadas en su capacidad de desarrollo e innovación tecnológica, y en la obtención y uso sistemático de los medios tecnológicos y organizacionales; b) las empresas obtienen congruencia organizacional y método para los esfuerzos de desarrollo tecnológico, de incorporación de tecnologías distintivas, y de innovación tecnológica, que llevan a cabo en sus procesos de creación, transformación y entrega de valor a clientes y consumidores; y, c) se complementa el esfuerzo organizacional que las empresas realizan para agregar valor a sus productos o servicios. Para desarrollar tales capacidades de desarrollo tecnológico e innovación, sistematizar los esfuerzos organizacionales con método y dirección, y organizar el diseño, ejecución y evaluación de proyectos tecnológicos, las empresas llevan a cabo una serie de tareas, acciones o procesos de gestión de tecnología que en conjunto podemos denominar como prácticas de gestión de tecnología (Sousa \& Kruglianskas, 1994), aunque otros autores les llaman herramientas de gestión de la tecnología (Fundación Cotec, 1999) o actividades que forman parte de procesos de gestión de tecnología (Probert, Phaal, \& Farrukh, 2000, p. 314). 


\subsection{OBJETIVO Y METODOLOGÍA UTILIZADA}

Con el objetivo de identificar los rasgos relevantes, así como las principales prácticas de gestión de tecnología de empresas innovadoras en México, que han ganado el Premio Nacional de Tecnología (PNT) de 1999 a 2007 se llevó a cabo un estudio exploratorio para analizar las experiencias de gestión de tecnología de 25 empresas. Para la realización del trabajo se realizó el análisis documental de las prácticas y resultados de gestión de tecnología que han reportado tales empresas a la oficina del PNT, se llevaron a cabo varias entrevistas con responsables de gestión de tecnología de diversas empresas, y además se compararon sus prácticas de gestión tecnológica con las características más relevantes de la gestión de tecnología que han sido reportadas en la literatura.

\section{RASGOS RELEVANTES DE LA GESTIÓN TECNOLÓGICA}

\subsection{CONDICIONES ORGANIZACIONALES PARA LA INNOVACIÓN}

A partir del análisis de la literatura especializada se identificaron un conjunto de rasgos relevantes de la gestión de tecnología en empresas. Estos rasgos significativos se muestran sintetizados en la Tabla 1. De ellos, un conjunto inicial tiene que ver con la generación de condiciones organizacionales necesarias para la innovación, a saber:

a) Una estrategia tecnológica definida (Burgelman, Christensen, \& Wheelwright, 2004; Clarke, Ford, \& Saren, 1989; Ford, 1988; Nelson, 1991; Pavitt, 1990).

b) Capacidad de respuesta a la demanda del mercado, o bien de creación de la misma (Marquis, 1969; Pavitt, 2003).

c) La empresa cuenta con una estructura organizacional especializada o enfocada en la gestión de tecnología (Abernathy \& Utterback, 1978; Nelson, 1991).

d) Capacidades esenciales para la creación y mantenimiento del know-how, de investigación, desarrollo e innovación ( $\mathrm{I}+\mathrm{D}+\mathrm{i})$, y de dominio de los activos complementarios, solos o de forma expandida (Goldbrunner, Hauser, List, \& Veldhoen, 2005; Grindley \& Sullivan, 1998; Hamel \& Prahalad, 1995; Pavitt, 2003; Roberts \& Frohman, 1978; Teece, 1986).

e) Un pool de procesos o prácticas sistematizadas de gestión de tecnología que garantiza que las cosas se hagan bien (Farrukh et al., 2004; Fundación Cotec, 1999; Fundación Premio Nacional 
de Tecnología, 2005; Menke, 1997; Omta, Boute, \& Engelen, 1994; Phaal, Paterson, \& Probert, 2006; Smith, 2003).

f) Una cultura organizacional que soporte la innovación (Goldbrunner et al., 2005; Husain, Sushil, \& Pathak, 2002; Lafley, 2008; Tushman \& O’Reilly III, 2004).

\subsection{GESTIÓN DE TECNOLOGÍA Y PROCESOS DE INNOVACIÓN}

Un segundo grupo de rasgos relevantes se relaciona con el rol que juega la gestión de tecnología en los procesos de innovación tecnológica, en la medida que: a) establece un marco de relaciones entre tecnología y capacidades empresariales (Christensen \& Overdorf, 2000); b) genera capacidades gerenciales para integrar equipos de trabajo que garanticen la implantación comercial de las innovaciones (Burgelman et al., 2004; Pavitt, 1990); c) aporta habilidades y competencias para responder a necesidades de mercados y clientes aprovechando oportunidades tecnológicas que se generan dentro y fuera de la organización con el fin de transformar el desempeño de productos, procesos, negocios y organizaciones (Marquis, 1969; Pavitt, 1990; Phaal et al., 1998); d) aporta una perspectiva estratégica al rol que juegan las tecnologías, y el impacto del cambio tecnológico, en el rumbo de los negocios (Burgelman et al., 2004; Clark et al., 1989; Ford, 1988; Nelson, 1991; Pavitt, 1990; Roberts, 1996), y, e) aporta un enfoque y sistematización en la realización de las tareas que le corresponden (Fundación Premio Nacional de Tecnología, 2005).

Estos rasgos relevantes identificados en la literatura publicada sobre gestión de tecnología se utilizaron para ser comparados con la información generada, y reportada, por 25 empresas innovadoras, de diversos tamaños y sectores, que han sido ganadoras del Premio Nacional de Tecnología (PNT) de México de 1999 a 2007. En la siguiente sección se analizan los resultados de dicha comparación.

\section{Rasgos relevantes}

\section{Condiciones organizacionales para la innovación}

La gestión de tecnología aporta un enfoque estratégico al manejo de los recursos tecnológicos de la empresa. Esto es, la empresa muestra una estrategia tecnológica claramente definida.

Gracias a la gestión de tecnología la empresa cuenta con la capacidad para el cuestionamiento continuo de la congruencia o idoneidad de los mercados.

La empresa cuenta dentro de su estructura organizacional con un área especializada o enfocada en la gestión de tecnología.

La empresa ha generado capacidades esenciales para la creación y mantenimiento del know-how, de I+D e innovación, y de dominio de los activos complementarios, sola o de forma expandida. 
La empresa cuenta con un pool de procesos o prácticas de gestión de tecnología que garantiza que "las cosas se hagan bien", que se hagan de forma efectiva.

Existe una cultura de innovación que justifica y soporta la gestión de tecnología en la empresa.

\section{Gestión de tecnología y procesos de innovación}

Las prácticas de gestión de tecnología establecen un marco de relaciones entre la tecnología, las capacidades de la empresa y los objetivos del negocio.

La empresa cuenta con la capacidad para orquestar e integrar grupos funcionales y de especialistas para la implantación de las innovaciones.

La empresa cuenta con habilidades para la explotación de las oportunidades tecnológicas (capacidad de innovación), y esto le aporta valor a la empresa.

La gestión de tecnología es responsabilidad de un profesional experimentado que forma parte del máximo comité de dirección de la empresa, alguien capaz de unir la tecnología con la estrategia corporativa global.

La gestión de tecnología está sistematizada y tiene atributos claramente definidos.

\section{Cuadro 1 - Rasgos relevantes de la gestión de tecnología}

Fuente: Elaborada por el autor.

\section{ANÁLISIS DE RESULTADOS}

En este trabajo se revisó la experiencia en gestión tecnológica reportada por 25 empresas ubicadas en México, de diversos tamaños y sectores, ganadoras del Premio Nacional de Tecnología entre 1999 y 2007. Los objetivos de dicho estudio fueron: i) identificar las características distintivas de la gestión de tecnología de un conjunto de empresas innovadoras en México, que han ganado en diferentes años el PNT, pero para los fines de este trabajo solo se comentan aquellas características que tienen que ver con los rasgos relevantes encontrados en la literatura; e, ii) identificar cuáles son las prácticas de gestión de tecnología más utilizadas por tales empresas. Una vez hechos los análisis particulares, se revisaron los elementos que tienen en común y sus diferencias más significativas. Se consideró que el análisis de 25 casos de empresas ganadoras del PNT, de un total de 35 casos publicados hasta el momento del análisis, era un número significativo que permitía revisar con certeza sus rasgos relevantes. Las empresas analizadas, el año en que ganaron el PNT, su tamaño y tipo, se indican en Cuadro 2.

\begin{tabular}{|c|l|c|l|}
\hline No. & \multicolumn{1}{|c|}{ Empresa } & Año & \multicolumn{1}{|c|}{ Tamaño } \\
\hline 1 & Probiomed & 1999 & Pequeña Industrial \\
\hline 2 & Laboratorios Sophia & 2000 & Mediana Industrial \\
\hline 3 & Vitro Automotriz & 2001 & Grande Industrial \\
\hline 4 & Centro de Investigación y Desarrollo Tecnológico (CID)- & 2001 & Grande de Servicios \\
\hline 5 & AMECE & 2001 & Pequeña de Servicios \\
\hline 6 & Delphi Automotive Systems & 2002 & Grande Industrial \\
\hline
\end{tabular}

Revista de Administração e Inovação, São Paulo, v. 7, n. 3, p. 58-78, jul./set. 2010 


\begin{tabular}{|c|l|c|l|}
\hline 7 & Condumex & 2002 & Grande Industrial \\
\hline 8 & Resirene & 2002 & Mediana Industrial \\
\hline 9 & Mabe & 2003 & Grande Industrial \\
\hline 10 & Centro de Ingeniería Avanzada en Turbomáquinas (CIAT) & 2003 & Grande de Servicios \\
\hline 11 & Orto de México & 2003 & Mediana Industrial \\
\hline 12 & Servicios, Construcciones y Montajes (Sercom) & 2003 & Pequeña de Servicios \\
\hline 13 & Grupo Cementos de Chihuahua (GCC) & 2004 & Grande Industrial \\
\hline 14 & Pfizer México & 2004 & Grande de Servicios \\
\hline 15 & Arroba Ingeniería & 2004 & Pequeña Industrial \\
\hline 16 & Instituto Bioclon & 2005 & Mediana Industrial \\
\hline 17 & Sony de Tijuana Este & 2006 & Grande Industrial \\
\hline 18 & Laboratorios Cryopharma & 2006 & Mediana Industrial \\
\hline 19 & Provista & 2006 & Pequeña Industrial \\
\hline 20 & Comnet & 2006 & Pequeña de Servicios \\
\hline 21 & Chrysler de México & 2007 & Grande Industrial \\
\hline 22 & Cidec Carso & 2007 & Grande de Servicios \\
\hline 23 & Nucitec & 2007 & Mediana Industrial \\
\hline 24 & Centro de Investigación en Polímeros (CIP) - COMEX & 2007 & Mediana de Servicios \\
\hline 25 & Grupo SETI & 2007 & Pequeña de Servicios \\
\hline
\end{tabular}

Cuadro 2 - Organizaciones analizadas en el estudio exploratorio

Fuente: Elaborada por el autor.

\subsection{RASGOS RELEVANTES DE LA GESTIÓN TECNOLÓGICA EMPRESARIAL}

Se aprovechó el estudio exploratorio para revisar los rasgos más relevantes de gestión de tecnología de las empresas que han ganado el Premio Nacional de Tecnología. Se confrontaron los hallazgos con lo reportado en la literatura especializada en gestión de tecnología. Una síntesis de la información obtenida se muestra en Cuadro 3, donde se indica la frecuencia encontrada de condiciones organizacionales para la innovación y de rasgos relacionados con el rol que juega la gestión de tecnología en los procesos de innovación, de acuerdo con la información proporcionada, y divulgada en la mayoría de los casos, por las propias empresas. En dicha tabla se presentan los rasgos relevantes de gestión tecnológica de mayor a menor frecuencia, agrupados en ambos grupos.

\begin{tabular}{|l|c|c|}
\hline \multicolumn{1}{|c|}{ Rasgos relevantes } & \multicolumn{2}{c|}{ Empresas que los reportan } \\
\hline I. Condiciones organizacionales para la innovación & Número & \% \\
\hline $\begin{array}{l}\text { La empresa ha generado capacidades esenciales para la creación y } \\
\text { mantenimiento del know-how, de I+D e innovación, y de dominio de los } \\
\text { activos complementarios. }\end{array}$ & 23 & 92 \\
\hline $\begin{array}{l}\text { Gracias a la gestión de tecnología la empresa cuenta con la capacidad para } \\
\text { el análisis a fondo de los mercados. }\end{array}$ & 20 & 70 \\
\hline La empresa cuenta con un pool de procesos o prácticas de gestión de & 18 & 72 \\
\hline
\end{tabular}

Revista de Administração e Inovação, São Paulo, v. 7, n. 3, p. 58-78, jul./set. 2010 


\begin{tabular}{|l|c|c|}
\hline tecnología. & 15 & 60 \\
\hline $\begin{array}{l}\text { Existe una cultura de innovación que justifica y soporta la gestión de } \\
\text { tecnología en la empresa. }\end{array}$ & 10 & 40 \\
\hline $\begin{array}{l}\text { La gestión de tecnología aporta un enfoque estratégico al manejo de los } \\
\text { recursos tecnológicos de la empresa. }\end{array}$ & 8 & 32 \\
\hline $\begin{array}{l}\text { La empresa cuenta dentro de su estructura organizacional con un área } \\
\text { especializada o enfocada en la gestión de tecnología. }\end{array}$ & 25 & 100 \\
\hline II. Gestión de tecnología y procesos de innovación & 23 & 92 \\
\hline $\begin{array}{l}\text { La empresa cuenta con habilidades para la explotación de las oportunidades } \\
\text { tecnológicas (capacidad de innovación). }\end{array}$ & 15 & 60 \\
\hline $\begin{array}{l}\text { La empresa es capaz de integrar grupos funcionales y de especialistas para } \\
\text { la implantación de las innovaciones. }\end{array}$ & 14 & 56 \\
\hline $\begin{array}{l}\text { Las prácticas de gestión de tecnología establecen un marco de relaciones } \\
\text { entre tecnología, capacidades y objetivos del negocio. }\end{array}$ & 7 & 28 \\
\hline $\begin{array}{l}\text { La gestión de tecnología es responsabilidad de un profesional } \\
\text { experimentado que forma parte del máximo comité de dirección. }\end{array}$ & \\
\hline La gestión de tecnología en la empresa tiene atributos claramente definidos. & 7 \\
\hline
\end{tabular}

Cuadro 3 - Frecuencia de rasgos relevantes en empresas analizadas

Fuente: Elaborada por el autor.

\subsection{CONDICIONES ORGANIZACIONALES PARA LA INNOVACIÓN}

En relación a los rasgos relevantes que constituyen condiciones organizacionales para la innovación en las empresas estudiadas, resaltan la construcción de capacidades esenciales, el desarrollo de procesos o prácticas de gestión tecnológica, el enfoque estratégico tecnológico que aporta y la presencia de prácticas culturales que soportan tanto la innovación como su gestión. Así, como se muestra en Cuadro 3, de las 25 empresas analizadas 23 (un 92\%) cuentan con capacidades esenciales para la creación de know-how, de I+D+i, y de dominio de activos complementarios para poder producir nuevos productos y procesos. Como se puede observar en Cuadro 4, que muestra los rasgos relevantes identificados en las empresas grandes industriales y de servicios estudiadas, se puede concluir que las empresas innovadoras prestan mucha atención a contar con una serie de capacidades esenciales para la realización de proyectos de investigación, desarrollo e innovación de producto y proceso, fortaleciendo sus capacidades de escalamiento, transferencia interna de tecnología, producción y comercialización de nuevos productos, y que eso ha ido aparejado con el desarrollo de procesos, técnicas y herramientas de gestión de tecnología -como planeación tecnológica, protección intelectual, vinculación con universidades y centros de I+D, vigilancia tecnológica, administración de proyectos tecnológicos- que les permite cumplir sus objetivos estratégicos y operacionales. 
Por otro lado, resalta el hecho de que un $80 \%$ de las empresas analizadas tengan capacidad para responder a clientes y mercados con los que trabajan. Para ello, la mayoría cuenta con procesos de vigilancia tecnológica (88\%), elaboración de estudios que les permite conocer estratégicamente sus mercados y clientes (72\%), y en menor medida actividades de benchmarking (44\%) como se muestra en Cuadro 7 que se comentará con más detalle en el apartado 3.2 de este artículo. Así, en relación con ello, el personal gerencial de un $72 \%$ de las empresas estudiadas dice contar con un conjunto (pool) de prácticas de gestión de tecnología que las hace efectivas, en términos de innovación. Como ya se mencionó, estas prácticas se comentarán más delante.

Con menor frecuencia, pero sin perder su importancia, el 60\% de las empresas (15) manifiestan contar con una cultura organizacional que favorece la innovación y en la que los líderes juegan un rol central, donde la gestión de tecnología permite conjuntar las capacidades operativas de la empresa con los objetivos de negocio y con la alineación de las tecnologías a lo largo y ancho de la empresa. Sin embargo, esto no es evidente en las empresas pequeñas, como se observa en Cuaro 5, que da cuenta de algunos rasgos culturales reportados por el personal de las empresas analizadas.

\begin{tabular}{|c|c|}
\hline Empresas grandes & Análisis resumido \\
\hline Vitro Automotriz & $\begin{array}{l}\text { Evidenciado por las tecnologías desarrolladas para la fabricación de nuevos } \\
\text { productos y componentes en la industria automotriz. Reporta una serie de sistemas } \\
\text { tecnológicos (sistemas de información y alerta tecnológica), procesos tecnológicos } \\
\text { (de I+DT, de innovación de productos y procesos, desarrollo de infraestructura, de } \\
\text { inteligencia de tecnologías, transferencia de tecnologías, protección intelectual, } \\
\text { planeación tecnológica, mejora continua de procesos y productos, solución de } \\
\text { problemas especiales y crónicos de la operación, APQP), y metodologías de trabajo } \\
\text { (definición de especificaciones técnicas, ejecución y administración de proyectos } \\
\text { tecnológicos, sistemas de calidad, gestión de recursos humanos, modelo de } \\
\text { evaluación de competencias). }\end{array}$ \\
\hline CID-Girsa & $\begin{array}{l}\text { Nuevos productos para las empresas del grupo industrial al que pertenecen. Cuentan } \\
\text { con capacidades propias, y con lo que denominan una Red de colaboración con } \\
\text { centros públicos de I+D. Durante un tiempo el CID se especializó en el segmento de } \\
\text { innovaciones mayores, distintivas o radicales, pero también colabora con sus } \\
\text { clientes en proyectos de innovación gradual. A sus clientes les ofrece servicios de } \\
\text { I\&D tecnológico, pero también investigación científica cuyos resultados son } \\
\text { patentables. }\end{array}$ \\
\hline Delphi & $\begin{array}{l}\text { Como lo demuestra su capacidad de patentamiento y de innovación de producto. } \\
\text { Involucran a sus proveedores tecnológicos para selección de materiales durante el } \\
\text { proceso de diseño; en diseño para manufactura (procesos, diseño de componentes, } \\
\text { tolerancias); y la introducción de nuevas tecnologías de materiales, procesos y } \\
\text { productos. }\end{array}$ \\
\hline Cidec Carso & $\begin{array}{l}\text { Evidenciado por su capacidad de I+D+i, y los } 104 \text { proyectos de innovación de } \\
\text { proceso ejecutados de } 2004 \text { a } 2007 \text { y } 82 \text { nuevos producto lanzados al mercado en el } \\
\text { mismo periodo. Desde } 1987 \text { comenzaron a vincularse con la UNAM y otras } \\
\text { universidades, ejecutando proyectos de I+D+i, asistencia técnica, transferencia, } \\
\text { entre otros. }\end{array}$ \\
\hline
\end{tabular}

Revista de Administração e Inovação, São Paulo, v. 7, n. 3, p. 58-78, jul./set. 2010 


\begin{tabular}{|c|c|}
\hline Mabe & $\begin{array}{l}\text { Como lo muestran los nuevos productos lanzados al mercado en México y } \\
\text { Sudamérica, la optimización de su tiempo de desarrollo de un proyecto (17 semanas) } \\
\text { y de un producto (de } 36 \text { a } 18 \text { meses, gracias al CAD/CAE), el uso de } 6 \text { Sigma, y sus } \\
\text { alianzas estratégicas. Su proceso de Introducción de Nuevos Productos es la } \\
\text { columna vertebral de todas las operaciones tecnológicas de Mabe. Es una } \\
\text { metodología de seguimiento y control de proyectos que consta de } 8 \text { etapas: 1) } \\
\text { alcance, 2) factibilidad, 3) definición, 4) confirmación, 5) pre-piloto, 6) piloto, 7) } \\
\text { producción, 8) post-producción. }\end{array}$ \\
\hline $\begin{array}{l}\text { Grupo Cementos de } \\
\text { Chihuahua }\end{array}$ & $\begin{array}{l}\text { Sobre todo la mejora y optimización de procesos de producción y de atención a } \\
\text { clientes; su capacidad de mantenimiento y uso del know-how de producción de } \\
\text { cemento; y prácticas de conocimiento de mercados y clientes, planeación } \\
\text { tecnológica, control de proyectos, protección vía marcas, y vinculación con centros } \\
\text { de I+D. }\end{array}$ \\
\hline Sony de Tijuana Este & $\begin{array}{l}\text { Ejemplos de ello son: el desarrollo de aplicaciones de integración de procesos de la } \\
\text { cadena de suministros a través de soluciones electrónicas de manejo de información } \\
\text { en tiempo real; diseño de nuevos conceptos de manufactura desarrollando equipos } \\
\text { de soldadura selectiva de alta precisión; desarrollo de software y hardware de alto } \\
\text { nivel para equipos de prueba y ajuste, con aplicaciones de comunicación e } \\
\text { incorporación de datos al producto. }\end{array}$ \\
\hline Chrysler de México & $\begin{array}{l}\text { Como lo muestran los ingresos obtenidos por la venta de nuevos productos: } 13.45 \% \\
\text { en } 2006 \text {. } \\
\text { Ejemplo de capacidades son los proyectos de implantación que reportan: a) creación } \\
\text { del Centro Avanzado de Ingeniería Automotriz; b) proyecto integral de ingeniería } \\
\text { para el desarrollo de una planta de ensamble automotriz de alta tecnología (cabina } \\
\text { extendida); c) desarrollo de una nueva plataforma para un nuevo modelo (camión } \\
\text { mediano Saltillo) y d) centro de Desarrollo de Soluciones de Software. }\end{array}$ \\
\hline
\end{tabular}

\section{Cuadro 4 - Capacidades de innovación tecnológica en empresas grandes}

Fuente: Elaborada por el autor.

\begin{tabular}{|l|l|l|l|}
\hline \multicolumn{2}{|c|}{$\begin{array}{l}\text { Empresas } \\
\text { estudiadas }\end{array}$} & \multicolumn{1}{|c|}{ Tipo } & \multicolumn{1}{c|}{ Análisis resumido } \\
\hline 1 & Probiomed & $\begin{array}{l}\text { Pequeña } \\
\text { Industrial }\end{array}$ & $\begin{array}{l}\text { Se capacita al personal en el manejo de tecnologías de punta y } \\
\text { procedimientos avanzados de gestión de la calidad. Con ello buscan } \\
\text { formar aptitudes, generar expectativas y enfocarse a resultados. }\end{array}$ \\
\hline 2 & $\begin{array}{l}\text { Laboratorios } \\
\text { Sophia }\end{array}$ & $\begin{array}{l}\text { Mediana } \\
\text { Industrial }\end{array}$ & No se reportó por la empresa. \\
\hline 3 & $\begin{array}{l}\text { Vitro } \\
\text { Automotriz }\end{array}$ & $\begin{array}{l}\text { Grande } \\
\text { Industrial } \\
\text { "La gestión de tecnología fundamenta, apoya la planeación } \\
\text { estratégica". "Somos seguidores competitivos. Nos hemos adecuado } \\
\text { tecnológicamente para poder competir en tiempos adecuados". } \\
\text { "Nuestra labor es agregar funciones al vidrio" (Arroyo, 2004). }\end{array}$ \\
\hline 4 & CID -GIRSA & $\begin{array}{l}\text { Grande de } \\
\text { Servicios }\end{array}$ & $\begin{array}{l}\text { Se incluía en las declaraciones del Grupo DESC al que pertenecía el } \\
\text { CID. Ahora forma parte del Grupo KUO. }\end{array}$ \\
\hline 5 & AMECE & $\begin{array}{l}\text { Pequeña de } \\
\text { Servicios }\end{array}$ & $\begin{array}{l}\text { Organismo de la iniciativa privada, liderado por las grandes cadenas } \\
\text { comerciales de México, que tiene como objetivo incrementar la } \\
\text { productividad y competitividad de las empresas a través del uso de } \\
\text { herramientas tecnológicas basadas en estándares internacionales. }\end{array}$ \\
\hline 6 & $\begin{array}{l}\text { Delphi } \\
\text { Automotive } \\
\text { Systems }\end{array}$ & $\begin{array}{l}\text { Grande } \\
\text { Industrial } \\
\text { directivos no tienen aversión al riesgo e invierten en I+D, así como por } \\
\text { la cartera de proyectos que ejecutan. }\end{array}$ \\
\hline
\end{tabular}

Revista de Administração e Inovação, São Paulo, v. 7, n. 3, p. 58-78, jul./set. 2010 


\begin{tabular}{|c|c|c|c|}
\hline 7 & Condumex $^{1}$ & $\begin{array}{l}\text { Grande } \\
\text { Industrial }\end{array}$ & $\begin{array}{l}\text { Se fomenta un ambiente propicio a la I+D e innovación. La cartera de } \\
\text { proyectos que ejecutaban tenía que producir innovaciones. }\end{array}$ \\
\hline 8 & Resirene & $\begin{array}{l}\text { Mediana } \\
\text { Industrial }\end{array}$ & No se describe explícitamente en su reporte. \\
\hline 9 & Mabe & $\begin{array}{l}\text { Grande } \\
\text { Industrial }\end{array}$ & $\begin{array}{l}\text { Cuentan con un Premio a la Creatividad e Innovación Tecnológica } \\
\text { instaurado en } 2001 .\end{array}$ \\
\hline 10 & CIAT & $\begin{array}{l}\text { Grande de } \\
\text { Servicios }\end{array}$ & $\begin{array}{l}\text { Se promueven actividades de fomento a la creatividad, el desarrollo de } \\
\text { ideas y el estímulo a los trabajadores: Innovation @ work (dos } \\
\text { concursos anuales), The Book of ideas (base de datos), Tinkering } \\
\text { Room; y el Día del Ingeniero (reconocimiento y premiación a los } \\
\text { mejores trabajos de ingeniería del año). }\end{array}$ \\
\hline 11 & Orto de México & $\begin{array}{l}\text { Mediana } \\
\text { Industrial }\end{array}$ & $\begin{array}{l}\text { En algunos casos se estimula la creatividad del personal con bonos } \\
\text { especiales. Dan libertad a su personal clave para proponer proyectos } \\
\text { nuevos que sean de su especial interés. Consideraban que un ambiente } \\
\text { de trabajo cómodo y agradable estimula la innovación. }\end{array}$ \\
\hline 12 & Sercom & $\begin{array}{l}\text { Pequeña de } \\
\text { Servicios }\end{array}$ & No lo reportan. \\
\hline 13 & GCC & $\begin{array}{l}\text { Grande } \\
\text { Industrial }\end{array}$ & No lo reportan. \\
\hline 14 & Pfizer México & $\begin{array}{l}\text { Grande de } \\
\text { Servicios }\end{array}$ & Incorporan los valores que la empresa promueve a nivel corporativo. \\
\hline 15 & $\begin{array}{l}\text { Arroba } \\
\text { Ingeniería }\end{array}$ & $\begin{array}{l}\text { Pequeña } \\
\text { Industrial }\end{array}$ & $\begin{array}{l}\text { El director general es el principal impulsor del lanzamiento al mercado } \\
\text { de productos mejorados. }\end{array}$ \\
\hline 16 & Instituto Bioclon & $\begin{array}{l}\text { Mediana } \\
\text { Industrial }\end{array}$ & La cultura de innovación está incorporada en su filosofía empresarial. \\
\hline 17 & Sony de Tijuana & $\begin{array}{l}\text { Grande } \\
\text { Industrial }\end{array}$ & $\begin{array}{l}\text { No lo precisan, pero afirman contar con una cultura de innovación a } \\
\text { nivel global y local. }\end{array}$ \\
\hline 18 & $\begin{array}{l}\text { Laboratorios } \\
\text { Cryopharma }\end{array}$ & $\begin{array}{l}\text { Mediana } \\
\text { Industrial }\end{array}$ & $\begin{array}{l}\text { El Director General se asegura de generar una cultura tecnológica en } \\
\text { torno a una visión compartida. }\end{array}$ \\
\hline 19 & Provista & $\begin{array}{l}\text { Pequeña } \\
\text { Industrial }\end{array}$ & No es evidente de sus reportes e información que proporcionan. \\
\hline 20 & Comnet & $\begin{array}{l}\text { Pequeña de } \\
\text { Servicios }\end{array}$ & $\begin{array}{l}\text { Según información de la empresa, está soportada en los valores } \\
\text { siguientes: innovación, integridad, calidad, trabajo en equipo, } \\
\text { crecimiento, rentabilidad. }\end{array}$ \\
\hline 21 & $\begin{array}{l}\text { Chrysler de } \\
\text { México }\end{array}$ & $\begin{array}{l}\text { Grande } \\
\text { Industrial }\end{array}$ & Se insiste en ello a nivel corporativo sobre todo. \\
\hline 22 & Cidec Carso & $\begin{array}{l}\text { Grande de } \\
\text { Servicios }\end{array}$ & $\begin{array}{l}\text { Como parte de la alineación de tecnologías, se fomenta un ambiente } \\
\text { propicio a la innovación mediante: Reportes de evaluación previa, } \\
\text { tesis, proyectos, nuevos negocios, Foro Cidec, conferencias } \\
\text { impartidas, vinculación con universidades e institutos de investigación. }\end{array}$ \\
\hline 23 & Nucitec & $\begin{array}{l}\text { Mediana } \\
\text { Industrial }\end{array}$ & Se resalta en su enfoque estratégico. \\
\hline 24 & CIP - Comex & $\begin{array}{l}\text { Mediana de } \\
\text { Servicios }\end{array}$ & No se reporta. \\
\hline 25 & Grupo Seti & $\begin{array}{l}\text { Pequeña de } \\
\text { Servicios }\end{array}$ & $\begin{array}{l}\text { Cultura de innovación en proceso de creación. Uno de los dos socios } \\
\text { de la empresa es el Director de Tecnología, principal impulsor del } \\
\text { desarrollo de nuevos productos. }\end{array}$ \\
\hline
\end{tabular}

Cuadro 5 - Rasgos culturales favorables a la innovación en empresas estudiadas

Fuente: Elaborada por el autor.

\footnotetext{
${ }^{1}$ La mayor parte de las acciones de Condumex fue adquirida por el grupo Carso en 1992, transformándose en la base de su división industrial, a la cual pertenece Cidec Carso. Es la única organización que ha ganado el PNT en dos ocasiones.

Revista de Administração e Inovação, São Paulo, v. 7, n. 3, p. 58-78, jul./set. 2010
} 
En quinto término se encontró con el hecho de que un $88 \%$ de las empresas estudiadas realizan planeación estratégica, y definen estrategias de negocios. Sin embargo, como se indica en Cuadro 3, solo 10 de las 25 empresas (un 40\%) exponen claramente su estrategia tecnológica, y 11 de ellas (un 44\%) reportan prácticas desarrolladas de gestión de cartera de proyectos tecnológicos, una actividad que forma parte del proceso de planeación tecnológica pero que sorprendentemente se realiza muchas veces sin haber delineado antes claramente una estrategia tecnológica.

Ahora bien, salta a la vista que hay aspectos organizacionales que están lejos de ser relevantes para las empresas analizadas. Por ejemplo, fue evidente la falta de estructuración organizacional de la gestión de tecnología en las organizaciones innovadoras: solo un 32\% de las empresas reportan un área responsable de las actividades de gestión tecnológica en el organigrama. Esto contradice lo señalado por los teóricos del diseño organizacional que plantean que una vez definida la estrategia de negocios, y las correspondientes estrategias funcionales, se debe delinear una estructura organizacional ad hoc para la puesta en práctica de dichas estrategias, en particular la tecnológica.

\section{GESTIÓN DE TECNOLOGÍA Y PROCESOS DE INNOVACIÓN}

Por otra parte, del conjunto de rasgos de gestión tecnológica que incide directamente en los procesos de innovación de las empresas destacan la habilidad para explotar oportunidades tecnológicas, la integración de equipos de trabajo para la innovación, y la alineación entre negocios, capacidades y tecnología. Así, como se indicó en Cuadro 3 la totalidad de las empresas analizadas muestran capacidad de explotación de las oportunidades tecnológicas, evidenciada por los nuevos productos y familias de productos que introducen al mercado, su capacidad de mejora de procesos, con base en su capacidad de desarrollo de tecnologías y de plataformas tecnológicas ${ }^{2}$. Esto se evidencia también en Cuadro 4 para el caso de las empresas grandes.

Un segundo aspecto a resaltar es la capacidad que la mayoría de las empresas estudiadas (un 92\%) reportan la integración de grupos de trabajo para llevar a cabo los procesos de innovación. Estos equipos adoptan formas diversas: multifuncionales, grupos de trabajo, grupos de investigación, por proyecto, consejos de $\mathrm{I}+\mathrm{D}$, comités de tecnología, de desarrollo de producto, entre otros, como se

\footnotetext{
${ }^{2}$ No hay que olvidar que son empresas innovadoras las aquí analizadas. Lo que varía entre ellas de forma notable es el tipo y número de prácticas de gestión tecnológica que utilizan.

Revista de Administração e Inovação, São Paulo, v. 7, n. 3, p. 58-78, jul./set. 2010
} 
observa en Cuadro 6, que muestra lo que reportan las pequeñas y medianas empresas industriales y de servicios que se analizaron.

\begin{tabular}{|l|l|}
\hline \multicolumn{1}{|c|}{ Empresas medianas } & \multicolumn{1}{c|}{ Análisis resumido } \\
\hline Laboratorios Sophia & $\begin{array}{l}\text { Cuenta, por ejemplo, con el Comité de Nuevos Productos que está integrado por } \\
\text { el Director General, el Vicepresidente, y los Directores de Finanzas, Comercial, } \\
\text { Científico y de Operaciones. }\end{array}$ \\
\hline Resirene & $\begin{array}{l}\text { Grupo de trabajo para innovación de equipo y proceso, con apoyo de asesores } \\
\text { externos (outsourcing tecnológico) para aumentar capacidades tecnológicas. }\end{array}$ \\
\hline Orto de México & Grupos de ingeniería de proyectos. \\
\hline Instituto Bioclon & $\begin{array}{l}\text { Equipos de I+D, con asesoría de expertos de universidades, por ejemplo del } \\
\text { Instituto de Biotecnología de la UNAM. }\end{array}$ \\
\hline Laboratorios Cryopharma & $\begin{array}{l}\text { Integración de equipos de trabajo multidisciplinarios durante la instalación de } \\
\text { equipo e infraestructura por parte del fabricante. }\end{array}$ \\
\hline Nucitec & Comité de proyectos. \\
\hline CIP-Comex & $\begin{array}{l}\text { Los investigadores de las distintas áreas trabajan en grupos multidisciplinarios } \\
\text { desarrollando proyectos en los campos de la investigación básica, investigación } \\
\text { aplicada y en el desarrollo de nuevos productos y procesos. }\end{array}$ \\
\hline Amece & $\begin{array}{l}\text { En su ámbito de acción, demuestran capacidad de desarrollo de nuevos servicios, } \\
\text { y de coordinación de esfuerzos con sus asociados para el impulso de nuevos } \\
\text { estándares. }\end{array}$ \\
\hline Sercom & Reportan un área de sistemas. \\
\hline Arroba Ingeniería & En el diseño de sus productos participan todas las áreas de la organización. \\
\hline Provista & Integran equipos de desarrollo de productos. \\
\hline Comnet & Cuentan con un Comité de tecnología en la empresa. \\
\hline Grupo Seti & Trabajan con sus clientes en la implantación de innovaciones. \\
\hline
\end{tabular}

Cuadro 6 - Integración de equipos para la innovación en empresas medianas y pequeñas

Fuente: Elaborada por el autor.

Y al igual que el rasgo anterior, un $60 \%$ de las empresas analizadas cuentan con prácticas de gestión de tecnología que establecen un marco de relaciones entre tecnología, capacidades y objetivos del negocio.

Llama también la atención de que solo un poco más de la mitad de las empresas estudiadas (un $56 \%$ ) cuenta con un directivo o gerente, con experiencia en el campo, que se encargue de coordinar y se responsabilice de los procesos de gestión de tecnología en ellas. Aún en las empresas innovadoras mexicanas, el rol de un gerente de tecnología o de innovación no ha sido asumido a cabalidad por la mayoría de las organizaciones, y esto se refleja en la carencia de definiciones más precisas en las estrategias tecnológicas que se plantean, por ejemplo. Es necesario realizar más investigaciones y estudios de caso que permitan conocer mejor cómo las empresas gestionan sus tecnologías y procesos de innovación. 


\subsection{PRÁCTICAS MÁS UTILIZADAS DE GESTIÓN DE TECNOLOGÍA}

Con el fin de identificar los prácticas o actividades de gestión de tecnología más frecuentes que utilizan las empresas estudiadas, sobre todo aquellas que han tenido más incidencia en sus actividades de innovación, se identificaron las prácticas que reportan cada una de las empresas estudiadas, y posteriormente se hizo un análisis comparativo de prácticas de gestión de tecnología de la totalidad de las empresas, dando como resultado Cuadro 7 que se muestra en la siguiente página. Las empresas están agrupadas por categorías: empresas industriales grandes, medianas y pequeñas; y empresas de servicios grandes, medianas (MS) y pequeñas. Las prácticas de gestión de tecnología identificadas en la Tabla 7 fueron reportadas en los casos publicados por la oficina del $\mathrm{PNT}^{3}$.

No se incluyen en esta tabla las prácticas reportadas de gestión de personal tecnológico y de gestión financiera por considerarse que no son de gestión de tecnología. Sí se incluyen algunas prácticas que no son propiamente de gestión de tecnología (planeación estratégica, gestión de la calidad, elaboración de estudios de mercados y clientes) pero que eran solicitadas por el PNT en sus convocatorias de 1999 a 2004.

Si bien las prácticas reportadas están determinadas por la información que se les solicita a las empresas en la Guía de participación del PNT, puede observarse la existencia de un cierto número de prácticas que comparten la mayoría de las empresas. Como se muestra en Cuadro 7, un 100\% de las empresas realizan actividades de protección intelectual. El $88 \%$ de las empresas, sin importar su tamaño o sector, realiza planeación tecnológica y lleva a cabo actividades de I+D Un $84 \%$ hace vigilancia tecnológica ${ }^{4}$ y ejecutan actividades de innovación de producto o servicio, y realizan planeación estratégica. Un $76 \%$ reporta actividades de vinculación con universidades y centros de I+D, y un $72 \%$ transferencia de tecnología, sobre todo dentro de la propia empresa, y conoce estratégicamente sus mercados y clientes. También, un 56\% de las empresas adquiere tecnología para completar sus capacidades de I+D+i, de producción y comercialización.

Hay que considerar, no obstante, que las empresas analizadas reportaron sus prácticas de gestión de tecnología en años diferentes, entre 1999 y 2007, y que, seguramente, la mayoría de las organizaciones ha evolucionado en forma de gestionar tecnología. De modo que lo que aquí se expone solo presenta una imagen estática de las prácticas de gestión de tecnología de estas empresas en dicho periodo. Sin embargo, si tomamos por ejemplo las 9 empresas ganadoras del PNT en los años 2006 y

\footnotetext{
${ }^{3}$ Se ratificó la información confrontando la información con personal de la oficina del PNT mediante entrevistas. En algunos casos se completó con información adicional obtenida de entrevistas con personal de empresas y documentos adicionales revisados.

${ }^{4}$ La vigilancia tecnológica recibe varios nombres por el personal gerencial de las empresas: identificación tecnológica, inteligencia tecnológica, inteligencia competitiva, vigilancia del entorno tecnológico, alerta tecnológica, monitoreo tecnológico, y de manera muy específica: monitoreo normativo.

Revista de Administração e Inovação, São Paulo, v. 7, n. 3, p. 58-78, jul./set. 2010
} 
2007 e identificamos sus prácticas de gestión de tecnología, encontramos también que el 100\% de ellas realiza actividades de protección intelectual, de I+D y de vigilancia tecnológica; y que el $89 \%$ lleva a cabo prácticas de planeación tecnológica, alineación de la gestión de tecnología, adquisición de tecnología y transferencia de tecnología. Un $78 \%$ de las empresas enmarca sus actividades dentro la planeación estratégica y realiza innovación de producto y servicios; y un $67 \%$ se vincula con universidades y centros de I+D, escalan sus desarrollos tecnológicos, asimilan tecnología y cuentan con sistemas y prácticas de gestión de calidad. Lo que muestra, en términos generales, que hay mucha coincidencia en las principales prácticas reportadas.

Si analizamos por tamaños, encontramos que de un total de 360 prácticas reportadas por la totalidad de las empresas analizadas, un $49.17 \%$ (177) de ellas fueron reportadas por empresas grandes industriales y de servicios, un 27.5\% (99) fueron reportadas por empresas medianas, y un $23.3 \%$ (84) por empresas pequeñas, lo cual suena lógico pues las grandes y medianas tienen mayores necesidades que atender, pero también más recursos para responder. Se puede observar también que conforme se incrementa el número de prácticas, éstas tienden a bajar en número, dado que son utilizadas solo por unas cuantas empresas. Las últimas doce prácticas, por ejemplo, son utilizadas por no más de tres empresas, posiblemente por tratarse de prácticas muy especializadas (Planeación avanzada de la calidad, Planes de desarrollo multigeneracional, por ejemplo), muy poco conocidas (Modelo de evaluación previa, planeación normativa), o cuyo dominio implica recursos y competencias de no fácil acceso (Prospectiva tecnológica, por ejemplo). Sin embargo, llama la atención encontrar empresas de diversos tamaños con número similares de prácticas reportadas, lo cual puede ser un indicador de que las empresas pequeñas y medianas están haciendo un esfuerzo de gestión tecnológica importante a pesar de contar con menos recursos que las grandes, y por el contrario que algunas empresas grandes están dejando de hacer cosas que podrían beneficiarlas. Así, por ejemplo, encontramos empresas pequeñas con 17, 15 y 14 prácticas reportadas ${ }^{5}$, medianas con 22, 16 y 16 prácticas (Bioclon, CIP y Nucitec, respectivamente), por contraste, grandes empresas con solo 9 y 12 prácticas reportadas.

\footnotetext{
${ }^{5}$ La primera es Probiomed que cuenta con 17 prácticas reportadas de gestión de tecnología, era en 1999 una empresa pequeña, ahora es una empresa grande con ventas anuales superiores a los 40 millones de dólares (Uribe, 2004). La empresa pequeña que reporta 15 prácticas es Arroba Ingeniería. Sin embargo, cabe señalar que el Director y socio responsable de la mayoría de las actividades de gestión de tecnología de Arroba Ingeniería dejó la empresa en 2005, lo cual seguramente ha redundado en una disminución de las mismas. La tercera con 14 prácticas reportadas es la empresa química Provista.

Revista de Administração e Inovação, São Paulo, v. 7, n. 3, p. 58-78, jul./set. 2010
} 


\begin{tabular}{|c|c|c|c|c|c|c|c|c|c|c|c|c|c|c|c|c|c|c|c|c|c|c|c|c|c|c|c|}
\hline & & \multicolumn{7}{|c|}{ Grandes Industriales } & \multicolumn{4}{|c|}{$\begin{array}{l}\text { Grandes de } \\
\text { Servicios }\end{array}$} & \multicolumn{6}{|c|}{ Medianas Industriales } & \multirow{2}{*}{ MS } & \multicolumn{3}{|c|}{$\begin{array}{l}\text { Pequeñas } \\
\text { Industriales }\end{array}$} & \multicolumn{4}{|c|}{$\begin{array}{l}\text { Pequeñas de } \\
\text { Servicios }\end{array}$} & \\
\hline No. & $\begin{array}{l}\text { Nombre de la práctica } \\
\text { de gestión tecnológica }\end{array}$ & A & B & $\mathrm{C}$ & D & E & F & G & $\mathrm{H}$ & I & $\mathbf{J}$ & K & $\mathrm{L}$ & $\mathbf{M}$ & $\mathbf{N}$ & 0 & $\mathbf{P}$ & Q & & $\mathrm{S}$ & $\mathbf{T}$ & $\mathrm{U}$ & V & W & $\mathbf{X}$ & $\mathbf{Y}$ & \\
\hline 1 & Protección intelectual. & $\boldsymbol{\sigma}$ & $\nabla$ & $\boldsymbol{\sigma}$ & - & - & $\nabla$ & 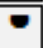 & - & - & - & $\bar{\sigma}$ & - & $\boldsymbol{\nabla}$ & - & $\boldsymbol{\sigma}$ & $\boldsymbol{\sigma}$ & - & $\boldsymbol{\sigma}$ & $\sigma$ & - & - & - & $\boldsymbol{\nabla}$ & $\boldsymbol{\sigma}$ & $\boldsymbol{\sigma}$ & 25 \\
\hline 2 & Planeación tecnológica & 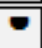 & $\nabla$ & $\nabla$ & - & $\nabla$ & 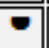 & - & - & & - & $\nabla$ & - & $\boldsymbol{\nabla}$ & - & - & $\sigma$ & 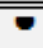 & $\bar{\sigma}$ & 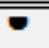 & $\nabla$ & $\boldsymbol{\nabla}$ & & $\boldsymbol{\nabla}$ & $\boldsymbol{\sigma}$ & & 22 \\
\hline 3 & I+D de Tecnología & $\sigma$ & $\overline{0}$ & $\bar{\sigma}$ & $\bar{\sigma}$ & $\bar{\sigma}$ & $\overline{0}$ & $\bar{\sigma}$ & $\bar{\sigma}$ & & $\bar{\sigma}$ & $\bar{\sigma}$ & $\bar{\sigma}$ & $\overline{0}$ & $\bar{\sigma}$ & $\bar{\sigma}$ & $\bar{\sigma}$ & $\bar{\sigma}$ & $\bar{\sigma}$ & $\bar{\sigma}$ & $\bar{\sigma}$ & $\bar{\sigma}$ & & & $\bar{\sigma}$ & $\bar{\sigma}$ & 22 \\
\hline$\overline{4}$ & Vigiancia tecnológica & $\bar{\sigma}$ & & $\bar{\sigma}$ & $\bar{\nabla}$ & $\bar{\sigma}$ & $\bar{\sigma}$ & $\overline{7}$ & $\bar{\sigma}$ & & $\bar{\nabla}$ & $\bar{\sigma}$ & $\bar{\nabla}$ & $\bar{\nabla}$ & $\overline{7}$ & $\overline{0}$ & $\overline{0}$ & $\overline{0}$ & $\bar{\sigma}$ & $\bar{\sigma}$ & $\bar{\nabla}$ & $\bar{\sigma}$ & & & $\overline{0}$ & $\bar{\sigma}$ & $\overline{21}$ \\
\hline$\overline{5}$ & Planeación estratégica ${ }^{\circ}$ & $\bar{\nabla}$ & $\nabla$ & $\bar{\nabla}$ & $\bar{\sigma}$ & $\overline{-1}$ & $\nabla \mid$ & $\overline{-1}$ & $\bar{\sigma}$ & $\bar{\sigma}$ & $\bar{\sigma}$ & $\bar{\nabla}$ & & $\nabla$ & & $\bar{\sigma}$ & & $\bar{\sigma}$ & $\bar{\nabla}$ & $\bar{\sigma}$ & $\bar{\sigma}$ & $\bar{\sigma}$ & 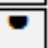 & $\boldsymbol{\nabla}$ & & $\bar{\sigma}$ & $\overline{21}$ \\
\hline$\overline{6}$ & $\begin{array}{l}\text { Innovación de producto } \\
\text { o servicio }\end{array}$ & - & • & $\boldsymbol{\bullet}$ & & • & - & & $\bullet$ & $\boldsymbol{\bullet}$ & $\boldsymbol{\bullet}$ & $\boldsymbol{\bullet}$ & $\boldsymbol{\bullet}$ & & $\boldsymbol{\bullet}$ & $\boldsymbol{\bullet}$ & $\boldsymbol{\bullet}$ & • & & - & - & - & • & • & - & $\boldsymbol{\bullet}$ & 21 \\
\hline 7 & Gestión de la calidad & $\sigma$ & 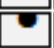 & $\bar{\sigma}$ & $\bar{\sigma}$ & $\bar{\sigma}$ & $\overline{-1}$ & $\bar{\sigma}$ & $\bar{\sigma}$ & $\bar{\sigma}$ & $\bar{\sigma}$ & $\bar{\sigma}$ & $\bar{\sigma}$ & $\overline{7}$ & & $\sigma$ & & $\bar{\sigma}$ & & $\bar{F}$ & $\bar{\sigma}$ & $\bar{\sigma}$ & & $\bar{\sigma}$ & & $\bar{\sigma}$ & 20 \\
\hline 8 & $\begin{array}{l}\text { Vinculación con } \\
\text { universidades y centros } \\
\text { deI+D }\end{array}$ & • & - & $\boldsymbol{\bullet}$ & $\boldsymbol{\bullet}$ & - & - & - & - & $\boldsymbol{\bullet}$ & $\boldsymbol{\bullet}$ & • & & - & & $\boldsymbol{\bullet}$ & & $\boldsymbol{\bullet}$ & - & - & $\boldsymbol{\bullet}$ & - & - & & & & 19 \\
\hline 9 & $\begin{array}{l}\text { Transferencia de } \\
\text { tecnologia }\end{array}$ & • & - & • & - & - & - & - & - & $\boldsymbol{\bullet}$ & - & $\boldsymbol{\bullet}$ & & & - & & $\boldsymbol{\sigma}$ & • & - & & & - & - & & - & & 18 \\
\hline 10 & $\begin{array}{l}\text { Conocimiento } \\
\text { estratégico e } \\
\text { integración de } \\
\text { mercados y clientes }\end{array}$ & - & - & - & $\boldsymbol{\bullet}$ & - & & & - & $\boldsymbol{\bullet}$ & - & - & & - & $\boldsymbol{\bullet}$ & $\boldsymbol{\bullet}$ & & • & & - & - & - & - & - & & & 18 \\
\hline 11 & $\begin{array}{l}\text { Adquisición de } \\
\text { tecnologia }\end{array}$ & & & - & & & $\bullet$ & - & & & & $\boldsymbol{\sigma}$ & - & - & & $\sigma$ & - & v & $\boldsymbol{v}$ & & & $\nabla$ & $\boldsymbol{v}$ & & $\boldsymbol{\bullet}$ & $\boldsymbol{\bullet}$ & 14 \\
\hline 12 & $\begin{array}{l}\text { Asimilación de } \\
\text { tecnología }\end{array}$ & & & $\boldsymbol{\sigma}$ & & - & - & & $\bullet$ & & - & $\bullet$ & & & & $\sigma$ & $\bullet$ & & - & & v & - & & • & - & - & 14 \\
\hline 13 & $\begin{array}{l}\text { Competitividad de } \\
\text { productos y procesos }\end{array}$ & - & • & $\boldsymbol{\bullet}$ & & - & & $\boldsymbol{\bullet}$ & • & $\boldsymbol{\bullet}$ & - & & & & - & $\boldsymbol{\bullet}$ & & & & & - & & & - & & & 12 \\
\hline 14 & $\begin{array}{l}\text { Administración de } \\
\text { proyectos tecnológicos }\end{array}$ & $\boldsymbol{\bullet}$ & - & • & & $\boldsymbol{\bullet}$ & & $\boldsymbol{v}$ & - & & - & & - & & & - & & $\boldsymbol{\bullet}$ & & - & - & & & & & & 12 \\
\hline 15 & Escalamiento & & $\overline{-7}$ & $\bar{\sigma}$ & & & & 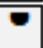 & $\bar{\sigma}$ & & & $\bar{\sigma}$ & $\bar{\sigma}$ & & & $\overline{0}$ & & $\bar{\sigma}$ & $\bar{\sigma}$ & $\bar{\sigma}$ & & $\bar{\sigma}$ & & & $\bar{\sigma}$ & & 12 \\
\hline 16 & Benchmarking & $\bar{\sigma}$ & & $\bar{\sigma}$ & & D & & & $\bar{\sigma}$ & & $\sigma$ & $\bar{\sigma}$ & & & $\sigma$ & $\bar{\sigma}$ & & $\bar{\sigma}$ & & & $\bar{\sigma}$ & & $\bar{\sigma}$ & & & & $\overline{\Pi 1}$ \\
\hline 17 & $\begin{array}{l}\text { Gestión de cartera de } \\
\text { proyectos tecnológicos }\end{array}$ & $\bullet$ & & & & & & - & $\boldsymbol{\bullet}$ & $\boldsymbol{\bullet}$ & & - & $\boldsymbol{\bullet}$ & & & - & & & - & - & - & & & & $\boldsymbol{\bullet}$ & & 11 \\
\hline 18 & Innovación de proceso & $\boldsymbol{\nabla}$ & $\bullet$ & $\boldsymbol{\sigma}$ & & & & $\bullet$ & $\boldsymbol{\sigma}$ & & & $\bar{\sigma}$ & & $\bullet$ & $\bullet$ & - & & - & $\boldsymbol{\sigma}$ & & & & & & & & 11 \\
\hline 19 & $\begin{array}{l}\text { Almeación de la gestión } \\
\text { de tecnología }\end{array}$ & & & & & & - & - & & & & - & & & & $\boldsymbol{\sigma}$ & - & & • & & & - & & & • & $\boldsymbol{\bullet}$ & 9 \\
\hline
\end{tabular}

${ }^{1}$ El modelo del PNT utilizado hasta el 2004 incluía criterios de planeación estratégica, conocimiento de mercados y gestión de calidad. Cuadro 7 - Prácticas de gestión de tecnología reportadas por las empresas estudiadas (continua) 


\section{Cuadro 7 - Prácticas de gestión de tecnología reportadas por las empresas estudiadas (continuación)}

\begin{tabular}{|c|c|c|c|c|c|c|c|c|c|c|c|c|c|c|c|c|c|c|c|c|c|c|c|c|c|c|c|}
\hline \multirow{2}{*}{ No } & \multirow{2}{*}{\begin{tabular}{|l|l} 
Nombre \\
de la \\
práctica \\
de gestión \\
tecnológic \\
a
\end{tabular}} & \multicolumn{7}{|c|}{ Grandes industriales } & \multicolumn{4}{|c|}{$\begin{array}{c}\text { Grandes de } \\
\text { servicios }\end{array}$} & \multicolumn{6}{|c|}{ Medianas industriales } & \multirow[t]{2}{*}{\begin{tabular}{|c|}
$\mathrm{M}$ \\
$\mathrm{S}$ \\
\end{tabular}} & \multicolumn{3}{|c|}{$\begin{array}{c}\text { Pequeñas } \\
\text { industriales }\end{array}$} & \multicolumn{4}{|c|}{$\begin{array}{c}\text { Pequeñas de } \\
\text { servicios }\end{array}$} & \multirow[b]{2}{*}{ Tota } \\
\hline & & $A$ & B & c & D & E & F & G & H & I & $\mathrm{J}$ & $\mathbf{K}$ & L & M & $\mathrm{N}$ & 0 & $\mathbf{P}$ & Q & & $s$ & $\mathrm{~T}$ & $\mathrm{U}$ & $\mathrm{V}$ & $\mathbf{w}$ & $\mathrm{x}$ & $\mathbf{Y}$ & \\
\hline 20 & $\begin{array}{l}\text { Administracio } \\
\text { n del } \\
\text { conocimiento }\end{array}$ & $\bullet$ & & & $\bullet$ & & & $\bullet$ & - & & & - & & & & - & & & $\bullet$ & & & & & & & $\bullet$ & 8 \\
\hline 21 & $\begin{array}{l}\text { Establecimien } \\
\text { to de } \\
\text { especificacion } \\
\text { es }\end{array}$ & & $\bullet$ & & & & & & $\bullet$ & & & & & & & & & & $\bullet$ & $\bullet$ & $\bullet$ & & & & - & & 7 \\
\hline 22 & $\begin{array}{l}\text { Evaluacion de } \\
\text { proyectos }\end{array}$ & & & $\bullet$ & & & & $\bullet$ & $\bullet$ & & & & & $\bullet$ & & & & & $\bullet$ & $\bullet$ & & & & & & & 6 \\
\hline 25 & $\begin{array}{l}\text { Plamagcion } \\
\text { avanzada de } \\
\text { la calidad }\end{array}$ & $\bullet$ & & $\bullet$ & & & & & & & & $\bullet$ & & & & & & & & & & & & & & & 3 \\
\hline 24 & $\begin{array}{l}\text { Auditona } \\
\text { tecnológica }\end{array}$ & & & & & & & & & & & & $\bullet$ & & & $\bullet$ & & & & $\bullet$ & & & & & & & 3 \\
\hline 25 & $\begin{array}{l}\text { Inventario } \\
\text { tecnológico }\end{array}$ & & & $\bullet$ & & & & & $\bullet$ & & & & & & & & & & & $\bullet$ & & & & & & & 3 \\
\hline 26 & $\begin{array}{l}\text { Seleccion } \\
\text { tecnologias }\end{array}$ & & & & & & & & & & & & $\bullet$ & $\bullet$ & & & & & & - & & & & & & & 3 \\
\hline 27 & $\begin{array}{l}\text { Dragnostico } \\
\text { tecnológico }\end{array}$ & & & & & - & & & $\bullet$ & & & & & & & $\bullet$ & & & & & & & & & & & 3 \\
\hline 28 & $\begin{array}{l}\text { Innovacion } \\
\text { organizaciona } \\
1\end{array}$ & & & & & & & & & & & $\bullet$ & & & & - & & & & & & & & & & & 2 \\
\hline 29 & $\begin{array}{l}\text { Prospectiva } \\
\text { tecnológica }\end{array}$ & & & & & & & & $\bullet$ & & & $\bullet$ & & & & & & & & & 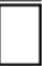 & & & & & & 2 \\
\hline 30 & $\begin{array}{l}\text { Modelo de } \\
\text { Evaluación } \\
\text { Previa }\end{array}$ & & & & & & & & & & & $\bullet$ & & & & & & & & & & & & & & & 1 \\
\hline 31 & $\begin{array}{l}\text { Plan de } \\
\text { desarrollo } \\
\text { multigeneraci } \\
\text { onal de } \\
\text { producto }\end{array}$ & & & & $\bullet$ & & & & & & & & & & & & & & & & & & & & & & 1 \\
\hline 32 & $\begin{array}{l}\text { Plan de } \\
\text { desarrollo } \\
\text { multigeneraci } \\
\text { onal de marca }\end{array}$ & & & & $\bullet$ & & & & & & & & & & & & & & & & & & & & & & 1 \\
\hline 33 & $\begin{array}{l}\text { Plan de } \\
\text { desarrollo } \\
\text { multigeneraci } \\
\text { onal de } \\
\text { tecnologia }\end{array}$ & & & & $\bullet$ & & & & & & & & & & & & & & & & & & & & & & 1 \\
\hline 34 & $\begin{array}{l}\text { Planeacion } \\
\text { nomativa }\end{array}$ & & & & & & & & 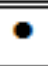 & & & & & & & & & & & & & & & & & & 1 \\
\hline & Empresas & $A$ & $\bar{B}$ & $\mathrm{c}$ & D & $E$ & F & $G$ & H & I & $\mathrm{J}$ & $\bar{K}$ & $\mathrm{~L}$ & II & $\mathrm{N}$ & 0 & $\mathbf{P}$ & 0 & $\overline{\mathbf{R}}$ & 5 & $\mathrm{~T}$ & U & $\mathrm{T}$ & $\overline{W W}$ & $\bar{x}$ & $\bar{Y}$ & \\
\hline & $\begin{array}{c}\text { Total de } \\
\text { prácticas por } \\
\text { empresa }\end{array}$ & 17 & 14 & 20 & 13 & 15 & 12 & 17 & 24 & 9 & 14 & 22 & 14 & 12 & 10 & 22 & 9 & 16 & $\begin{array}{l}1 \\
6\end{array}$ & $\begin{array}{l}1 \\
7\end{array}$ & $\begin{array}{l}1 \\
5\end{array}$ & 14 & 8 & 8 & 12 & 10 & 360 \\
\hline
\end{tabular}

Cuadro 7 - Prácticas de gestión de tecnología reportadas por las empresas estudiadas

Fuente: Elaborada por el autor.

En Cuadro 8 se muestran las 25 empresas analizadas (de 1999 a 2007) y el total de prácticas de gestión de tecnología que se identificaron en información proporcionada al Premio Nacional de Tecnología y casos publicados. La información se presenta de mayor a menor frecuencia de prácticas encontradas. 
Cabe hacer la aclaración de que a partir del año 2005 cambió el modelo de gestión de tecnología del PNT. En el nuevo modelo funcional se solicita información de 19 procesos de gestión de tecnología, y además se precisa qué información se tiene que reportar, lo cual permite evaluar qué tan sistematizados y maduras son sus prácticas. Temas como planeación estratégica, sistemas de calidad, patrimonio tecnológico ya no se solicitan a partir del modelo de 2005. Esto, sin duda, puede haber influido en el incremento de prácticas (procesos) de gestión de tecnología reportadas por las organizaciones a partir de ese año.

\begin{tabular}{|l|l|c|c|}
\hline \multicolumn{1}{|c|}{ Empresa } & Tamaño o categoría & Año & $\begin{array}{c}\text { Prácticas } \\
\text { reportadas }\end{array}$ \\
\hline $\begin{array}{l}\text { Centro de Investigación y Desarrollo Tecnológico } \\
\text { (CID) - Girsa }\end{array}$ & Grande de Servicios & 2001 & 24 \\
\hline Cidec Carso & & & 22 \\
\hline Instituto Bioclon & Grande de Servicios & 2007 & 22 \\
\hline Condumex & Mediana Industrial & 2005 & 20 \\
\hline Vitro Automotriz & Grande Industrial & 2002 & 17 \\
\hline Chrysler de México & Grande Industrial & 2001 & 17 \\
\hline Probiomed & Grande Industrial & 2007 & 17 \\
\hline Centro de Investigación en Polímeros & Pequeña Industrial & 1999 & 16 \\
\hline Nucitec & Mediana de Servicios & 2007 & 16 \\
\hline Grupo Cementos de Chihuahua & Mediana Industrial & 2007 & 15 \\
\hline Arroba Ingeniería & Grande Industrial & 2004 & 15 \\
\hline Pfizer México & Pequeña Industrial & 2004 & 14 \\
\hline Delphi Automotive Systems & Grande de Servicios & 2004 & 14 \\
\hline Laboratorios Sophia & Grande Industrial & 2002 & 14 \\
\hline Provista & Mediana Industrial & 2000 & 14 \\
\hline Mabe & Pequeña Industrial & 2006 & 13 \\
\hline Sony de Tijuana Este & Grande Industrial & 2003 & 12 \\
\hline Resirene & Grande Industrial & 2006 & 12 \\
\hline Comnet & Mediana Industrial & 2002 & 12 \\
\hline Orto de México & Pequeña de Servicios & 2006 & 10 \\
\hline Grupo SETI & Mediana Industrial & 2003 & 10 \\
\hline Centro de Ingeniería Avanzada en Turbomáquinas & Pequeña de Servicios & 2007 & 9 \\
\hline Laboratorios Cryopharma & Mediana Industrial & 2006 & 9 \\
\hline Servicios, Construcciones y Montajes (Sercom) & Pequeña de Servicios & 2003 & 8 \\
\hline $\begin{array}{l}\text { Asociación Mexicana de Estándares para el } \\
\text { Comercio Electrónico (Amece) }\end{array}$ & Pequeña de Servicios & 2001 & 8 \\
\hline Cuadro 8 Empresas ganadas & & \\
\hline
\end{tabular}

Cuadro 8 - Empresas ganadoras del PNT y sus prácticas de gestión tecnológica

Fuente: Elaborada por el autor. 


\section{CONCLUSIONES}

La investigación mostró que las actividades de gestión de tecnología que se realizan en las empresas analizadas cumplen con algunas características que poseen empresas innovadoras reportadas en la literatura (Pavitt, 1990): i) involucran la colaboración continua e intensiva y la interacción entre grupos funcionales y especializados, aún en empresas pequeñas como aquí se evidenció; ii) han mostrado capacidad de acumulación de conocimientos y capacidades tecnológicas; y, iii) son altamente diferenciadas. Queda por demostrar si las actividades que realizan de I+D son a tal grado inciertas que solo una parte pequeña de los resultados de sus proyectos de I+D tiene éxito comercial, para lo cual habría que investigar cómo se ejecutan y gestionan los proyectos de $\mathrm{I}+\mathrm{D}+\mathrm{i}$ en este tipo de empresas, algo que quedó fuera del alcance de este trabajo.

Las empresas estudiadas revelan que en su operación y actividades de innovación cumplen, si bien en forma diferenciada como se explicó antes, con los rasgos relevantes de gestión de tecnología que el autor identificó en el análisis de la literatura especializada sobre el tema, y que tanto el conjunto de rasgos que tiene que ver con la generación de condiciones necesarias para la innovación, como con el rol que juega la gestión de tecnología en los procesos de innovación tecnológica, son atendidos por empresas que hacen de la innovación uno de sus principales características.

El estudio reveló que, sin importar su tamaño o sector, las empresas son capaces de desarrollar capacidades de innovación tecnológica, evidenciada por los nuevos productos que introducen al mercado cada año, por su capacidad de optimización de procesos, y su capacidad de desarrollo de tecnologías y plataformas tecnológicas. Esto se vio reforzado por el hecho de que la gran mayoría de las empresas analizadas (92\%) cuentan con capacidades para la creación de know-how, de I+D+i, y de dominio de activos complementarios de producción y comercialización de nuevos productos.

La investigación permitió constatar que, a pesar de que el $60 \%$ de las empresas manifiestan contar con una cultura organizacional que favorece la innovación, esto no es del todo evidente en las empresas pequeñas, si bien en algunas de ellas el director general, o alguno de sus fundadores, es quien marca la pauta sobre el rumbo a seguir, el enfoque del negocio y los valores que imperan en la empresa. No está por demás recalcar que, éste análisis se basa exclusivamente en la información proporcionada por las empresas, y que puede haber casos donde éstas cuentan con un pool mayor de prácticas de gestión de tecnología que no están siendo consideradas aquí. Habrá que realizar estudios posteriores para identificar con mayor precisión la totalidad de las prácticas de gestión de tecnología que realizan, su frecuencia de uso, su grado de dominio y el impacto que tiene cada una de ellas sobre los procesos de innovación de la empresa. No obstante, la identificación de rasgos relevantes y 
prácticas permitió hacer una amplia exploración sobre las características de la gestión de tecnología de empresas innovadoras en el ámbito mexicano, y se espera que ello contribuya a un mejor entendimiento sobre los esfuerzos que éstas empresas realizan para mantenerse competitivas gracias a la innovación.

\section{REFERENCIAS}

Abernathy, W. J., \& Utterback, J. M. (1978). Patterns of industrial innovation. Technology Review, 80(7), 40-47.

Arroyo, M. (2004). Entrevista en las instalaciones de Vitro Automotriz (Testimonio a Enrique A. Medellín y Vicente Borja García. Monterrey, 12 de octubre.

Burgerlman, R. A., Christensen, C. M., \& Wheelwright, S. C. (2004). Strategic management of technology and innovation (4th ed.). New York: McGraw-Hill.

Clarke, K., Ford, D., \& Saren, M. (1989). Company technology strategy. R\&D Management, 19(3), 215-229.

Christensen, C. M., \& Overdorf, M. (2000). Meeting the challenge of disruptive change. Harvard Business Review, 78(2), 66-76.

Farrukh, C., Fraser, P., Hadjidakis, D., Phaal, R., Probert, D., \& Tainsh, D. (2004). Developing an integrated technology management process. Research Technology Management, 47(4), 39-46.

Ford, D. (1988). Develop your technology strategy. Long Range Planning, 21(5), 85-95.

Fundación Cotec. (1999). Pautas metodológicas en gestión de la tecnología y de la innovación para empresas - temaguide. Madrid: Autor.

Fundación Premio Nacional de Tecnología. (2005). Guía de participación 2005 del premio nacional de tecnología. México: Autor.

Goldbrunner, T., Hauser, R., List, G., \& Veldhoen, S. (2005). The four dimensions of intelligent innovation: winning the race for profitable growth. Munich: Booz Allen Hamilton.

Grindley, P., \& Sullivan, P. H. (1998). Irreconcilables differences? Managing the knowledge creation interfaces. In P. H. Sullivan. Profiting from intellectual capital: extracting value from innovation (pp. 84-101). New York: John Wiley \& Sons.

Hamel, G., \& Prahalad, C. K. (1995). Compitiendo por el futuro: estrategia crucial para crear los mercados del mañana. México: Ariel Sociedad Económica. 
Husain, Z., Sushil, \& Pathak, R. D. (2002). A technology management perspective on collaborations in the Indian automobile industry: a case study. Journal of Engineering and Technology Management, 19(2), 167-201.

Lafley, A. G. (2008, Aug. 26). P\&G’s Innovation Culture. Strategy+Business Magazine, pp. 1-10.

Marquis, D. G. (1969). The anatomy of successful innovations. Innovation, 1, 28-37.

Menke, M. M. (1997). Essentials of R\&D strategic excellence. Research Technology Management, $40(5), 42-47$.

Nelson, R. R. (1991). The role of firm differences in an evolutionary theory of technical advance. Science and Public Policy, 18(6), 347-352.

Omta, S. W. F., Boute, L. M., \& Engelen, J. M. L. (1994). Managing industrial pharmaceutical R\&D: a comparative study of management control and innovative effectiveness in European and AngloAmerican companies. $R \& D$ Management, 24(4), 303-315.

Pavitt, K. (2003). The process of innovation (SPRU Electronic Working Paper Series, 89). Brighton: University of Sussex.

Pavitt, K. (1990). What we know about the strategic management of technology. California Management Review, 32(3), 17-26.

Phaal, R., Paterson, C. J.m \& Probert, D. R. (1998). Technology management in manufacturing business: process and practical assessment. Technovation, 18(8/9), 541-553.

Probert, D. R., Phaal, R., \& Farrukh, C. J. P. (2000). Development of a structured approach to assessing technology management practice. Proceedings of the Institution of Mechanical Engineers, Part B: Journal of Engineering Manufacture, 214(4), 313-321.

Roberts, E. B. (1996). Ideas generales sobre la gestión de la innovación tecnológica. In Roberts, E.B., Gestión de la innovación tecnológica (Clásicos Cotec, n. 1) (pp. 53-77). Madrid: COTEC.

Roberts, E. B., \& Frohman, A. L. (1978). Strategies for improving research utilization. Technology Review, 80(5), 33-39.

Smith, R. D. (2003). The chief technology officer: strategic responsibilities and relationships. Research Technology Management, 46(4), 28-36.

Sousa, J. E. R., \& Kruglianskas, I. (1994). Práticas de gestão tecnológica e competitividade no setor de autopeças (pp. 703-726). Anais do Simpósio Nacional de Gestão da Inovação Tecnológica, 18. São Paulo: NPGT/USP. 
Teece, D. J. (1986). Profiting from technological innovation: implications for integration, collaboration, licensing and public policy. Research Policy, 15, 285-305.

Tushman, M. A., \& O’Reilly III, C. A. (2004). Ambidextrous organizations: managing evolutionary and revolutionary change. In R. A. Burgelman, C. M. Christensen, \& S. C. Wheelwright, Strategic management of technology and innovation (4th ed.) (pp. 925-956). New York: McGraw-Hill.

Uribe, J. (2004). Pasos firmes en la biotecnología. Recuperado em 12 de marzo, 2006, de http://www.dialogoejecutivo.com.mx/Ediciones/Agosto04/Entrevista\%20Agosto\% 2004.html.

\title{
MANAGEMENT OF TECHNOLOGY IN MEXICAN INNOVATIVE FIRMS
}

\begin{abstract}
This paper presents the experience of management of technology of 25 Mexican innovative firms from several sectors and sizes that had won the National Technology Award (NTA) between 1999 and 2007. The objective is to analyze their distinctive characteristics and the main practices of management of technology that the firms had reported. The methodology chosen consisted of an exploratory research, using information collected by the NTA, interviews with some managers and information published for the firms. The activities of innovation in these firms are in conformity with the characteristics of innovative firms reported in the specialized literature; and disclose that in their operation and practices of innovation fulfill the relevant characteristics found by the author in this literature. Two groups of relevant characteristics were identified: The first support the generation of necessary conditions for innovation, and the second are practices of management of technology that play an important role in the process of technology innovation. Both groups of distinctive characteristics are used, in different ways, for the firms that are competing based in innovation practices.
\end{abstract}

Keywords: Management of technology; Innovative firms; Distinctive characteristics; Mexico.

Data do recebimento do artigo: 05/06/2010

Data do aceite de publicação: 25/08/2010 